\title{
Vertical surface brightness profiles of boxy bulges
}

\section{G. Aronica ${ }^{1}$, M. Bureau ${ }^{2}$, E. Athanassoula ${ }^{3}$ and R. -J. Dettmar ${ }^{1}$}

${ }^{1}$ Astronomisches Institut der Ruhr-Universität Bochum, Bochum, D-44780, Germany email: aronica@astro.rub.de

${ }^{2}$ Sub-Department of Astrophysics, University of Oxford, Keble Road, Oxford, OX1 3RH, UK

${ }^{3}$ Laboratoire d'Astrophysique de Marseille, 2 place Le Verrier, 13248 Marseille Cedex 4, France

\begin{abstract}
The thickening of the bar in barred disk galaxies has a strong influence in shaping the morphology in the inner regions of a disk galaxy above the galactic plane. The result of such a secular evolutionary process can be observed in galaxies with box/peanut shaped (b/ps) bulges. We have applied a one-dimensional fitting method to our sample of 30 edge-on disk galaxies using different fitting function approaches. A clear increase in scale height can be observed in the area of the most prominent $\mathrm{b} / \mathrm{ps}$ isophotes compared to the neighbouring disk and bulge areas, in agreement with the predictions of the bar thickening model.
\end{abstract}

Keywords. infrared: galaxies, methods: data analysis, galaxies: photometry, galaxies: evolution, galaxies: structure, galaxies: spiral, galaxies: bulges, instabilities

\section{Box/peanut shaped bulges}

The work of Bureau \& Freeman (1999) showed that box/peanut shaped (b/ps) bulges can be observed frequently $(\approx 71 \%$ of their sample) in barred edge-on disk galaxies. That result and the work of Bureau, Aronica, Athanassoula, et al. (2006) corroborated the view of a stellar bar as the primary driver for the formation of the b/ps structure. In fact, those structures can evolve from a vertical instability in the bar (bar buckling, e.g., Pfenniger \& Friedli 1991) causing a vertical thickening of that component (e.g., Patsis, Skokos, \& Athanassoula 2002, Athanassoula 2005) on secular timescales.

\section{Analysis and results}

In order to analyze the vertical surface brightness distribution we used $K_{n}$-band near infrared data of the sample galaxies described in Bureau \& Freeman (1999) consisting of 30 galaxies. We have applied to the vertical surface brightness distribution of those galaxies a one-dimensional fitting approach based only on a thin disk component. The functions used were Sersic type functions, Gauss-Hermite polynomials, and the canonical exponential and sech type functions.

The innermost regions of the disk of galaxies with a b/ps structure are characterized by a global minimum in scale height (see Figure 1); a possible indication for a rather flat component like a compact cold disk. Further, the regions with the most prominent $\mathrm{b} / \mathrm{ps}$ isophotes show higher values for the scale height compared to the neighbouring disk and bulge areas. Also, the shape parameters of all fitting functions used show rather flat-topped vertical surface brightness distributions, contrary to the outer disk regions. Both changes, the local maxima in scale height and the flat-topped shaped distributions in the region with the most prominent b/ps isophotes, are consistent with the model of $\mathrm{b} / \mathrm{ps}$ bulge formation out of the thickening of the bar in those galaxies. 


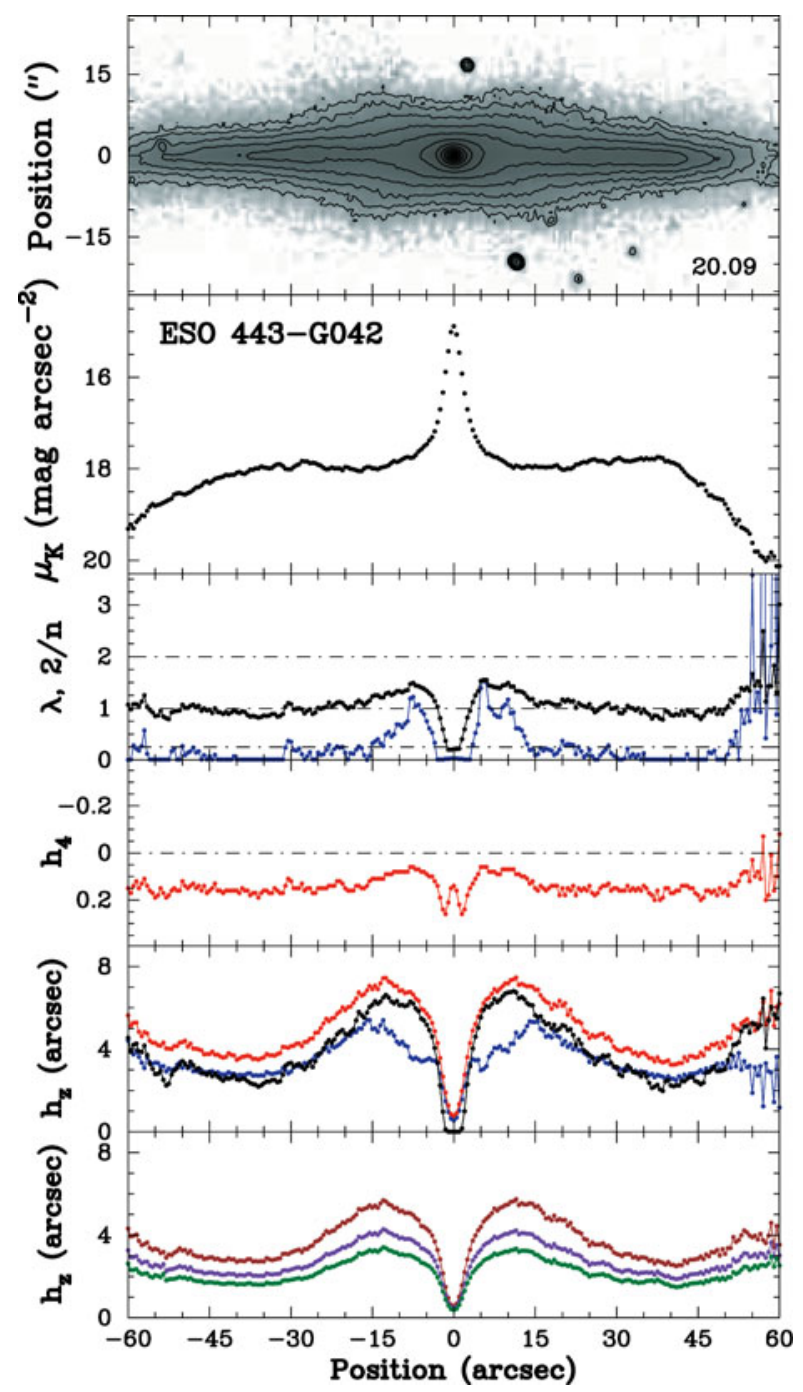

Figure 1. Results of the one-dimensional fitting approaches for the galaxy ESO 443-G042. In the first two panels, the grayscale $K_{n}$-band image with isocontours, respectively the radial surface brightness profile taken along the major axis of the galactic plane at $z=0$ are plotted. The next two panels show the results for the shape parameters of a Sersic type $(\lambda)$, a generalized sech type $(2 / n)$, and a Gauss-Hermite polynomial type $\left(h_{4}\right)$ fitting approach. All those parameters are a measure for the peakiness of the fitted distribution. In the last two panels the fitted scale height for all fitting functions used is displayed; the upper one shows the results for the functions with varying shape, whereas the lower one the results for fixed shapes (e.g. an exponential function).

\section{References}

Athanassoula, E. 2005, MNRAS 358, 1477

Bureau, M. \& Freeman, K. C. 1999, AJ 118, 2158

Bureau, M., Aronica, G., Athanassoula, E., Dettmar, R.-J., Bosma, A., \& Freeman, K. C. 2006, MNRAS 370, 753

Pfenniger, D. \& Friedli, D. 1991, A\& $A$ 252, 75

Patsis, P. A., Skokos, Ch., \& Athanassoula, E. 2002, MNRAS 337, 578 